\title{
Buchhalter versus Visionär
}

\section{Remo Osterwalder}

Dr. med., Vizepräsident der FMH, Departementsverantwortlicher Dienstleistungen und Berufsentwicklung

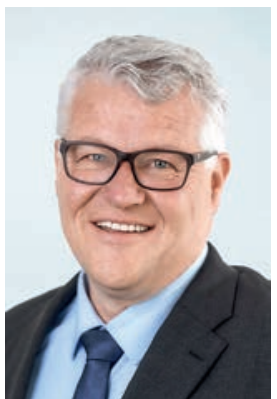

Zuerst muss klargestellt werden, dass ich für die Berufsgruppe der Buchhalter grossen Respekt habe, da ich ohne deren Hilfe zur Aufarbeitung und Analyse der Zahlen verloren wäre. Aber sie bzw. ihre Tätigkeiten illustrieren symbolisch die Situation der Ärztinnen und Ärzte im heutigen Gesundheitswesen. Erstere sind die Verwalter von Zahlen, welche sich auf den Ist-Zustand konzentrieren. Die Buchhalter analysieren die Vergangenheit - bis man die Zahlen interpretieren kann, ist schon einige Zeit vergangen. Es ist sicher richtig und wichtig, solche Analysen durchzuführen, wie uns der Bericht über unsere tägliche Arbeit des Kollegen Daniel Oertle aufzeigt [1]. Wir können auch feststellen, dass wir weiterhin Kolleginnen und Kollegen verlieren an Tätigkeiten ohne direkten Patientenkontakt. Sicher sollten wir wissen, weshalb es dazu kommt und was die jeweiligen Beweggründe sind.

Solche Analysen allein tragen jedoch noch nicht zur Lösung des Problems bei. Auf verschiedenen Stufen unseres Gesundheitssystems haben wir sehr viele Verwalter, welche sich profilieren und prominent auftreten. Wenn Gesundheitsökonom Stefan Felder eine

\section{Der Visionär stellt sich nicht nur die Zukunft vor, er gestaltet sie auch.}

Rationierung der medizinischen Leistungen wünscht, kommt dies einer Bankrotterklärung gleich: Es kann keine Lösung sein, dass man einfach den Zugang zur Gesundheitsversorgung verweigert. Dies führt direkt zur Zweiklassenmedizin und ermöglicht nur einer begrenzten Bevölkerungsschicht unbegrenzten $\mathrm{Zu}$ gang zu einer hochwertigen Gesundheitsversorgung. Wer dann auf der Strecke bleibt, ist schon heute klar. Wo aber sind die Anderen, die Visionäre in unserem Gesundheitssystem? Es gibt sie, wir treffen sie praktisch überall an: Es sind ärztliche Kolleginnen und Kol- legen, weitere Gesundheitsfachpersonen, aber auch Politiker und Kostenträger. Sie alle versuchen, mit gezielten und vorwiegend lokalen Projekten einen Beitrag zur Gesundheitsversorgung zu leisten. Was es braucht, ist der vernünftige Einsatz der vorhandenen Mittel. Hier kommt der Visionär zum Einsatz, welcher Modelle entwickelt, die den zukünftigen Anforderungen gerecht werden. So wird sich der Beruf des Arztes ändern: Wahrscheinlich arbeiten wir fortan mit Hilfe der Informatik vermehrt mit Algorithmen.

Studien als alleinige Behandlungsgrundlage reichen jedoch nicht aus, um alle Sektoren der Medizin real

\section{Visionäre Vorschläge sind nicht nur einseitige Lösungen, sondern sind für alle Akteure gleichermassen interessant.}

abzubilden. Wie wir alle wissen, erfüllt nicht jede Studie die Anforderung der grösstmöglichen Annäherung an den Idealzustand. Aber nicht nur wir Ärztinnen und Ärzte werden uns solcher Hilfsmittel bedienen, sondern vermehrt auch andere Berufsgruppen, welche Patienten im ambulanten Sektor versorgen. Einerseits soll daraus ein Effizienzgewinn entstehen, andererseits können so auch die vorhandenen Mittel zielgerechter eingesetzt werden. Ein Beispiel hierfür sind etwa die Choosing-Wisely-Listen der Schweizerischen Gesellschaft für Allgemeine Innere Medizin, welche in Algorithmen einfliessen könnten und damit vermehrt umgesetzt würden. Notwendig für solche Entwicklungen sind überzeugende Anreize sowohl für die Ärzteschaft als auch die Patienten. Die Anreize sind so zu gestalten, dass sie für alle Akteure interessant sind. Dies kann nur gemeinsam erfolgen, da einseitige Umsetzungen von Vorschlägen selten zu Konsens führen. \footnotetext{
Literatur

1 Oertle D. Die Hausärzte sterben aus, na und? Schweiz Ärztezeitung.
} 2016;97(51-52):1804-5. 